\title{
Fungitoxicidade dos extratos vegetais e do óleo essencial de Lippia gracilis Schauer sobre o fungo Monosporascus cannonballus Pollack e Uecker
}

\author{
Luciana Cristina Borges Fernandes ${ }^{1}$, Cynthia Cavalcanti de Albuquerque ${ }^{1}$, Rui Sales Júnior ${ }^{2}$, Francisco Fábio \\ Mesquita Oliveira ${ }^{1}$, Eleneide Pinto Gurgel ${ }^{1}$, Maria Valdiglêzia de Mesquita ${ }^{1}$, Mônica Danielle Sales da Silva ${ }^{1}$
}

${ }^{1}$ Universidade do Estado do Rio Grande do Norte - UERN, Avenida Professor Antônio Campos, s/n, CEP 59625-620, Mossoró, RN. ${ }^{2}$ Universidade Federal Rural do Semi-árido - UFERSA, BR-110 Km 47, Bairro Presidente Costa e Silva - Mossoró/RN. Apoio financeiro: CAPES.

Autora para correspondência: Luciana Cristiana Borges Fernandes (lucianacbf@hotmail.com)

Data de chegada: 07/02/2014. Aceito para publicação em: 03/03/2015.

$10.1590 / 0100-5405 / 1978$

\section{RESUMO}

Fernandes, L.C.B.; Albuquerque, C.C.; Sales Júnior, R.; Oliveira, F.F.M.; Gurgel, E.P.; Mesquita, M.V.; Silva, M.D.S. Fungitoxicidade dos extratos vegetais e do óleo essencial de Lippia gracilis Schauer sobre o fungo Monosporascus cannonballus Pollack e Uecker. Summa Phytopathologica, v.41, n.2, p.153-155, 2015.

Alecrim da Chapada (Lippia gracilis Schauer) é considerada uma planta rica em óleo essencial e possui atividade antimicrobiana comprovada, devido aos monoterpenos fenólicos carvacrol e timol. O objetivo deste trabalho foi avaliar o efeito in vitro do óleo essencial e dos extratos etanólicos de raízes e folhas de L. gracilis no controle do fungo Monosporasccus cannonballus, causador do colapso do meloeiro. O extratos foram testados nas concentrações de 2500,5000 e 7500 ppm e o óleo essencial de L. gracilis foi avaliado nas concentrações 255, 340 e 425 ppm tendo também um controle positivo com o fungicida comercial Captan ${ }^{\circledR} \mathrm{e}$ um negativo apenas com meio BDA (batata, dextrose, ágar). Discos de micélio com $3 \mathrm{~mm}$ de diâmetro foram inoculados no centro de placas de Petri e as medições do crescimento micelial do fungo foram realizadas 48 horas após a montagem do experimento. Verificou-se que o óleo essencial de L. gracilis nas três concentrações testadas foi eficiente para o controle do fungo, com percentuais de inibição de $100 \%$, em comparação ao tratamento com o controle negativo $(0 \%)$ que não apresentou inibição. $\mathrm{O}$ extrato radicular, nas concentrações de 5000 e 7500 ppm e o extrato foliar na concentração de 7500 ppm também proporcionaram um percentual de inibição de $100 \%$.

Palavras-chave: Alecrim da chapada, antimicrobiana, colapso do meloeiro

\section{ABSTRACT}

Fernandes, L.C.B.; Albuquerque, C.C.; Sales Júnior, R.; Oliveira, F.F.M.; Gurgel, E.P.; Mesquita, M.V.; Silva, M.D.S. Fungitoxicity of plant extracts and essential oil of Lippia gracilis Schauer on the fungus Monosporascus cannonballus Pollack and Uecker. Summa Phytopathologica, v.41, n.2, p.153-155, 2015.

"Alecrim da Chapada" (Lippia gracilis Schauer) is a plant which is considered to be rich in essential oil and proven to have antimicrobial activity due to the phenolic monoterpenes carvacrol and thymol. The aim of this study was to evaluate the in vitro effect of essential oil and ethanol extracts from roots and leaves of L. gracilis on the control of the fungus Monosporasccus cannonballus, the causal agent of melon collapse. The extracts were tested at the concentrations 2500,5000 and $7500 \mathrm{ppm}$ and L. gracilis essential oil was evaluated at the concentrations 255,340 and $425 \mathrm{ppm}$; there was also a positive control with the commercial fungicide $\operatorname{Captan}^{\circledR}$ and a negative control only with PDA medium (potato, dextrose, agar). Mycelium discs of $3 \mathrm{~mm}$ diameter were inoculated in the center of Petri plates and measurements of mycelial growth were performed at 48 hours after the establishment of the experiment. L. gracilis essential oil, at the three tested concentrations, was effective in controlling the fungus, showing inhibition percentages of $100 \%$, compared to the treatment with the negative control $(0 \%)$, which showed no inhibition. The root extract, at concentrations $5000 \mathrm{ppm}$ and $7500 \mathrm{ppm}$, and the leaf extract, at a concentration of $7500 \mathrm{ppm}$, also provided inhibition percentage of $100 \%$.

Additional keywords: Alecrim da Chapada, antimicrobial action, collapse of melon.

Monosporascus cannonballus Polack \& Uecker é um dos principais fitopatógenos envolvidos no colapso do meloeiro e um dos mais destrutivos dessa cultura no mundo, pois ataca a raiz e caracteriza-se por possuir ascósporos que permanecem no solo durante muito tempo (9).

Algumas tentativas de controle têm sido utilizadas, entretanto tem sido pouco eficientes, devido ao fato de tratar-se de um fungo termófilo e bem adaptado ao clima árido e semiárido. Uma das formas de manejo é o controle químico a base de fungicida e uso de outros fumigantes, de forma isolada ou em combinação com a solarização do solo (9). Todavia, o uso intensivo desses produtos tem causado diversos problemas de ordem ambiental, como a contaminação dos alimentos, do solo e da água (6). Assim, compostos naturais extraídos de algumas espécies vegetais, podem substituir produtos agrícolas convencionais, graças a sua potente atividade antimicrobiana (2).

O gênero Lippia (Verbenaceae) inclui aproximadamente 200 espécies de ervas, arbustos e de árvores de pequeno porte, e dentre essas espécies L. gracilis Schauer se destaca, devido à presença de óleo essencial com atividade antimicrobiana comprovada principalmente aos monoterpenos fenólicos timol e carvacrol, seus componentes marjoritários $(8,10)$.

Considerando que muitas das doenças causadas por patógenos radiculares não são eficientemente controladas por produtos químicos 
e que podem causar impactos ao ambiente, o presente trabalho teve como objetivo avaliar o efeito dos óleos essenciais e dos extratos vegetais de $L$. gracilis na inibição do crescimento micelial in vitro de M. cannonballus.

O experimento foi montado nos meses de novembro e dezembro de 2013 no Laboratório de Cultura de Tecidos Vegetais da Universidade do Estado do Rio Grande do Norte (UERN), Mossoró-RN. No experimento dos extratos, raízes e folhas de $L$. gracilis foram separadas, pesadas e secas em estufa com circulação forçada de ar por 72 horas. Em seguida, o material vegetal foi triturado separadamente e colocado em recipiente de vidro âmbar contendo $8 \mathrm{~L}$ de etanol a $70 \%$ para maceração sob agitação ocasional. O líquido extraído passou por uma primeira filtração em papel de filtro sobre pressão reduzida e uma segunda filtração por gravidade com funil fechado com algodão. $\mathrm{O}$ filtrado em seguida foi concentrado em rotaevaporador sob pressão reduzida à temperatura de $\pm 80^{\circ} \mathrm{C}$, para separação do etanol. A solução obtida foi colocada em banho Maria a uma temperatura constante de $65^{\circ} \mathrm{C}$ para evaporação da água e obtenção do material sólido. Em virtude da presença de forte espuma no extrato radicular de L. gracilis, foi feito um teste para saponinas, onde parte do material sólido do extrato foi pesada e dissolvida em $6 \mathrm{~mL}$ de água destilada e em seguida foi filtrada. A solução foi agitada fortemente por 3 minutos e observou-se a formação de espumas. A espuma persistente e abundante indicou a presença de saponinas.

Após esse procedimento, os extratos secos tanto de folha quanto de raiz foram diluídos para obtenção das concentrações testadas: 2500, 5000 e 7500 ppm. A diluição dos extratos foi realizada em meio BDA (batata, dextrose, ágar), o qual foi, em seguida, autoclavado por aproximadamente 15 minutos.

Além dos extratos, testou-se também o óleo essencial de L. gracilis, o qual foi extraído de folhas através do processo de hidrodestilação utilizando-se o aparato de Clevenger. Nesse caso, o óleo foi acrescentado ao meio BDA após a sua autoclavagem e resfriamento a $45{ }^{\circ} \mathrm{C}$. As concentrações avaliadas foram: 255, 340 e $425 \mathrm{ppm}$. Para termos de comparação, foi estabelecido um meio sem adição de extratos vegetais que serviu como controle negativo e um tratamento contendo o fungicida $\operatorname{Captan}^{\circledR}$ na concentração de 4075 ppm. Em câmara de fluxo laminar, $20 \mathrm{~mL}$ dos meios de cultura foram vertidos em placas de Petri de $100 \mathrm{~mm}$ de diâmetro. O experimento foi inteiramente casualizado (DIC) contendo 11 tratamentos e 4 repetições, com 3 placas para cada repetição, perfazendo um total de 132 placas, sendo 12 placas para cada tratamento.

Discos de micélio do fungo com aproximadamente $3 \mathrm{~mm}$ de diâmetro foram retirados de culturas com 5 dias em meio de BDA(batata, dextrose, ágar) e inoculados no centro de placas de Petri contendo os diferentes tratamentos. A variável avaliada foi o crescimento micelial, o qual foi medido através de medições diametralmente opostas com auxílio de um paquímetro, quando o tratamento controle atingiu o máximo de crescimento dentro dos limites da placa de Petri. Para determinar a percentagem de inibição, os dados do crescimento micelial individual de cada fungo foram usados, por meio da aplicação da fórmula adaptada de Edington et al. (4): $\mathrm{I}=[(\mathrm{CFC}-\mathrm{CFT}) / \mathrm{CFC}] \mathrm{x}$ 100, Onde: $\mathrm{I}=$ porcentagem de inibição; $\mathrm{CFC}=$ crescimento do fungo no controle; $\mathrm{CFT}=$ crescimento do fungo no tratamento.

Nos trabalhos in vitro, o óleo essencial em todas as concentrações avaliadas e os extratos foliar (7500 ppm) e radicular (5000 e 7500 ppm) inibiram $100 \%$ do crescimento micelial do fungo (Fig. 1). A inibição fúngica incitada por esses extratos naturais não diferiram estatisticamente do controle positivo (com fungicida comercial Captan $^{\circledR}$ ), porém quando comparados ao controle negativo (apenas meio de cultura) observou-se diferença significativa (Fig. 1). Sem dúvida alguma, os dados revelaram que o óleo essencial é mais eficiente quando comparado aos extratos, pois doses consideravelmente mais baixas já inibem $100 \%$ do crescimento fúngico.

A ação biológica do óleo contra os microrganismos se dá em virtude do carvacrol e o timol, compostos majoritários nessa espécie $(8,10)$ cujo mecanismo de ação já é bem conhecido, pois, em virtude da natureza lipofílica do óleo, há forte interação deste com a membrana plasmática da célula, resultando na formação de canais, através dos quais há extravasamento de conteúdo e consequente desorganização celular (3).

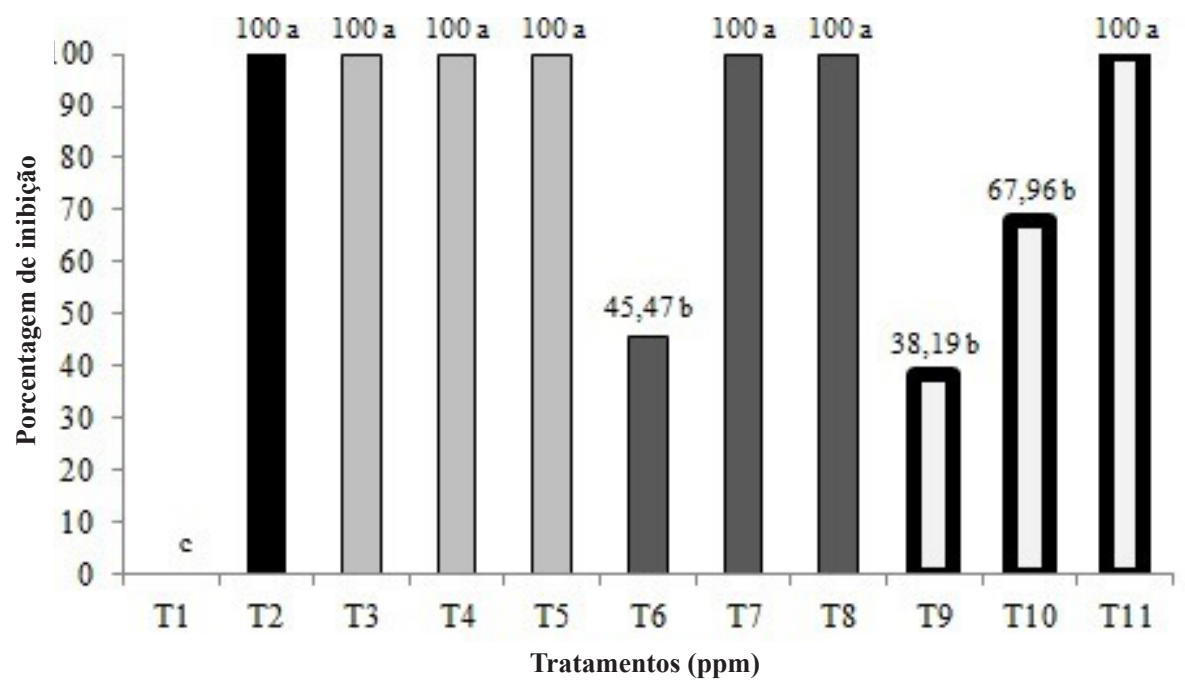

Figura 1. Percentagem de inibição do crescimento micelial in vitro de $M$. cannonballus pelo óleo essencial de $L$. gracilis. Os tratamentos avaliados foram: $T_{1}$ $(0 \mathrm{ppm}), \mathbf{T}_{2}$ (Fungicida comercial Captan $\left.{ }^{\circledR}\right), \mathbf{T}_{3}$ (Óleo essencial de L. gracilis na concentração de $255 \mathrm{ppm}$ ), $\mathbf{T}_{4}$ (Óleo essencial de $L$. gracilis na concentração de 340 ppm), $\mathbf{T}_{5}$ (Öleo essencial de L. gracilis na concentração de $425 \mathrm{ppm}$ ), $\mathbf{T}_{6}$ (Extrato radicular de L. gracilis na concentração de $2500 \mathrm{ppm}$ ), $\mathbf{T}_{7}$ (Extrato radicular de L. gracilis na concentração de $5000 \mathrm{ppm}$ ), $\mathbf{T}_{8}$ (Extrato radicular de L. gracilis na concentração de $7500 \mathrm{ppm}$ ), $\mathbf{T}_{9}$ (Extrato foliar de $L$. gracilis na concentração de $2500 \mathrm{ppm}$ ), $\mathbf{T}_{10}$ (Extrato foliar de L. gracilis na concentração de 5000 ppm), $\mathbf{T}_{11}$ (Extrato foliar de L. gracilis na concentração de 7500 ppm). UERN, Mossoró, RN, 2014. Letras minúsculas iguais nas barras não diferem entre si pelo teste de Tukey a $1 \%$ de probabilidade. CV\% $=14,73$. 
Nesse estudo, a atividade antifúngica do extrato radicular foi mais eficiente quando comparada ao foliar, pois doses de 5000 ppm já foram suficientes para inibir $100 \%$ do crescimento micelial, enquanto que nessa mesma concentração do extrato foliar, o fungo cresceu $32,04 \%$ (Fig. 1).

Outro aspecto importante diz respeito à presença de saponinas no extrato radicular de L. gracilis. Provavelmente, a presença de saponinas nas raízes de $L$. gracilis tenha neutralizado o crescimento micelial de M. cannonballus, uma vez que vários trabalhos corroboram com este resultado, evidenciando a sua atividade fungicida $(1,7)$. O mecanismo de ação das saponinas é semelhante ao do óleo, pois de acordo com Barile et al. (1), os compostos presentes nestas formam complexos com esteróis, resultando num aumento da permeabilidade da membrana do fungo e vazamento do conteúdo de suas células.

Pelo exposto, verificou-se a eficiência do óleo essencial e do extrato radicular de L. gracilis frente ao fungo M. cannonballus. Portanto, o presente estudo abre novas possibilidades para a indústria química no sentido de lançar no mercado novos produtos químicos de origem vegetal a fim de controlar fungos fitopatogênicos e que sejam menos danosos ao meio ambiente.

\section{AGRADECIMENTOS}

Os autores agradecem a Coordenação de Aperfeiçoamento de Pessoal de Nível Superior - CAPES

\section{REFERÊNCIAS BIBLIOGRÁFICAS}

1. Barile, E.; Bonanomi, G.; Antignani, V.; Zolfaghari, B.; Ebrahim Sajjadi, S.; Scala, F.; Lanzotti, V. Saponins from Allium minutiflorum with antifungal activity. Phytochemistry, Washington, v. 68, 596-603, 2007.

2. Benini, P.C.; Schwan-Estrada, K.R.F.; Klais, E.C.; Cruz, M.E.S.; Itak, A.T.; Mesquini, R.M.; Stangarlin, J.R.; Tolentino Júnior, J.B. Efeito in vitro do óleo essencial e extrato aquoso de Ocimum gratissimum colhido nas quatro estações do ano sobre fitopatógenos. Arquivo do Instituto Biológico, São Paulo, v. 77, n. 4, p. 677-683, 2010.

3. Costa, A.R.T.; Amaral, M.F.Z.J.; Martins, P.M.; Paula, J.A.M.; Fiuza, T.S.; Resvenzol, L.M.F.; Paula, J.R.; Bara, M.T.F. Ação do óleo essencial de Syzygium aromaticum (L.) Merr. \& L. M. Perry sobre as hifas de alguns fungos fitopatogênicos. Revista Brasileira de Plantas Medicinais, Paulínia, v. 13, n. 2, p. 240-245, 2011.

4. Edington, L.V.; Khew, K.L.; Barron, G.L. Fungitoxic spectrum of benzimidazole compounds. Phytopathology, Saint Paul, v. 61, n. 1, p. 42-44, 1971

5. Leme, M.I.S.; Camargo, M.; Furlani, A.C.F.A.; Panizzi, R.C.; Leite, R.F.; Rosa, J. Efeito in vitro de capim limão no desenvolvimento micelial de Colletotrichum acutatum. Summa Phytopathologica, Botucatu, v. 33, p. S92, 2007. Suplemento.

6. Morandi, M.A.B.; Bettiol, W. Controle biológico de doenças de plantas no Brasil. In: Bettiol, W.; Morandi, M.A.B. (Ed.). Biocontrole de doenças de plantas: Uso e Pespectivas. Jaguariúna: Embrapa Meio Ambiente, 2009. p. 7-14.

7. Saha, S.; Walia, S.; Kumar, J.; Parmar, B.S. Structure-biological activity relationships in triterpenic saponins: the relative activity of protobassic acid and its derivatives against plant pathogenic fungi. Pest Management Science, London, v. 66, p. 825-831, 2010.

8. Sarrazin, S.L.F.; Oliveira, R.B.; Barata, L.E.S.; Mourão, R.H.V. Chemical composition and antimicrobial activity of the essential oil of Lippia grandis Schauer (Verbenaceae) from the western Amazon. Food Chemistry, United Kingdon, v. 134, p. 147-1478, 2012.

9. Silva, K.J.P.; Cordeito, A.G.; Nogueira, D.R.S.; Sales Junior, R. Monosporascus cannonballus: Agente causal do colapso ou morte súbita do meloeiro. Revista Verde, Mossoró, v. 5, n. 4, p. 11-18, 2010.

10. Zare, Z.; Majd, A.; Sattari, T.N.; Iranbakhsh, A.; Mehrabian, S. Antimicrobial activity of leaf and flower extracts of Lippia nodiflora L. (Verbenacea). Journal of Plant Protection Research, Wielkopolskie, v. 52, n. 4, p. 401-403, 2012. 